\title{
Palliative Care Discussions in Multiple System Atrophy: A Retrospective Review
}

\author{
Anita M. Dayal, Mary E. Jenkins, Mandar S. Jog, Kurt Kimpinski, \\ Penny MacDonald, Teneille E. Gofton
}

\begin{abstract}
Objective: Multiple system atrophy (MSA) is an incurable neurodegenerative illness in which progressive symptoms, including stridor and acute laryngeal obstruction, occur. Advanced care planning and palliative care discussions in people living with MSA are not well defined. The aim of the present study is to evaluate advanced care planning and current practices in palliative care in MSA to identify opportunities for improving quality of care. Methods: The study is a retrospective chart review assessing the focus and timing of palliative care discussions in people living with MSA. Some 22 charts were reviewed. Results: A total of 22 patients were included. The most common symptoms were parkinsonism, orthostatic hypotension, GI/GU dysfunction, ataxia and gait impairment. Six patients had stridor. Of the palliative care discussions that took place, the most common topics were diagnosis, symptoms or symptom management, and prognosis. In the majority of patients who died and who had a do-not-attempt-resuscitation order, discussions surrounding resuscitation and goals of care took place only hours before death. Conclusions: There is no standard approach to advanced care planning and palliative care discussions in people living with MSA. We propose a framework to guide advanced care planning and palliative care discussions in MSA.
\end{abstract}

RÉSUMÉ: Examen rétrospectif des discussions abordant la question des soins palliatifs dans le cas de l'atrophie multi-systématisée. Objectif: L'atrophie multi-systématisée (AMS) une affection neuro-dégénérative incurable dont les symptômes (p. ex. : stridor, obstruction sévère du larynx, etc.) évoluent progressivement. La planification anticipée des soins palliatifs et la façon d'aborder ce sujet ne sont pas encore bien définies chez les patients qui en sont atteints. Cette étude vise ainsi à évaluer les pratiques actuelles et la planification anticipée en matière de soins palliatifs afin de déterminer les possibilités d'améliorer la qualité des soins leur étant prodigués. Méthodes: Reposant sur l'examen rétrospectif de 22 dossiers de patients, l'étude a tenté d'évaluer à quel moment et sur quoi ont porté les discussions menées avec eux. Résultats: Parmi les symptômes cliniques le plus souvent observés chez ces 22 patients, on peut noter les suivants : parkinsonisme, hypotension orthostatique, troubles gastro-intestinaux ou génito-urinaires, ataxie et démarche chancelante. Fait à noter, un stridor a été signalé parmi six d'entre eux. En ce qui concerne les discussions menées au sujet des soins palliatifs, l'établissement du diagnostic, les symptômes, leur soulagement ainsi que les pronostics ont été les sujets les plus fréquemment abordés. Pour une majorité de patients décédés qui avaient obtenu une ordonnance de non-réanimation, les discussions entourant la réanimation et la finalité des soins prodigués n'ont eu lieu que quelques heures avant le décès. Conclusions: Il n'existe pas d'approche standard en ce qui regarde la planification et les discussions portant sur les soins palliatifs offerts aux patients atteints de l'AMS. Nous avons ainsi voulu proposer un cadre de référence adapté à ces enjeux.

Keywords: movement disorders, palliative care, multiple system atrophy, end of life, advance care planning

doi:10.1017/cjn.2016.439

Can J Neurol Sci. 2017; 44: 276-282

\section{INTRODUCTION}

Multiple system atrophy (MSA) is a neurodegenerative disease of the central and autonomic nervous systems with a prevalence of 5 per $100,000 .{ }^{1}$ MSA is incurable, and treatment is sym ptomatic, which carries a poor prognosis and a mean survival of 7-10 years. $^{2}$ For people living with MSA, stridor is a major complication that develops in $30 \%$ of cases. ${ }^{2,3}$ While tracheostomy is only required in a minority of cases, ${ }^{4,5}$ respiratory failure arising from stridor may present acutely, and tracheostomy may be required as a life-supportive measure. For people living with MSA, there is a significant possibility that decisions about life-sustaining measures will be made in emergent situations. It is therefore important that people living with MSA and their caregivers have early and deliberate advance care planning discussions.
MSA is rare, and little evidence exists to guide physicians in their approach to care. ${ }^{5}$ This retrospective, non-concurrent cohort study explores the symptom burden of patients living with MSA and the palliative care discussions that take place throughout the disease trajectory. The aim of our study is to evaluate advance care planning and current practices in palliative care in MSA at a single centre in order to identify opportunities to improve quality of care.

From the Department of Clinical Neurological Sciences, Schulich School of Medicine and Dentistry, Western University, London, Ontario, Canada.

Received July 23, 2015. Final Revisions Submitted October 1, 2016. ACCEPTANCE OCTOBER 6, 2016.

Correspondence to: Teneille Gofton, London Health Sciences Centre, University Hospital, Western University, Room B2-223, 339 Windermere Road, London, Ontario, Canada N6A 5A5. Email: teneille.gofton@lhsc.on.ca. 
We also propose a framework for advance care planning and palliative care discussions in MSA.

\section{MethodS}

\section{Standard Protocol Approvals}

The study was approved by the Health Sciences Research Ethics Board at Western University. All data were de-identified prior to analysis.

\section{Inclusion and Exclusion Criteria}

Patients with a clinical diagnosis of MSA known to inpatient or outpatient neurology services at London Health Sciences Centre (London, Ontario, Canada) between January of 2004 and January of 2014 and meeting the criteria for a clinical diagnosis of probable $\mathrm{MSA}^{6}$ were included in our analysis. Patients were identified from the clinical practices of all movement disorder specialists at London Health Sciences Centre. Patients with parkinsonian syndromes of uncertain aetiology or with a diagnosis of an alternate Parkinson's Plus syndrome were excluded from the analysis.

\section{Data Collection}

Electronic medical records and paper charts for all visits to London Health Sciences Centre were reviewed by the authors (AD, TG). The data abstracted included the following: sex, age, age at diagnosis, duration of disease, deceased (if applicable), symptom burden including stridor and tracheostomy, evidence of a substitute decision maker or power of attorney for healthcare, evidence of palliative care discussions and content, presence of do-not-attempt-resuscitation (DNAR) orders, time from admission to death, time from palliative discussions to death and time from establishing DNAR to death.

\section{Data Analysis}

Data on common clinical symptoms were collected: ${ }^{6}$ orthostatic hypotension, dysarthria, ataxia, gait impairment, dysarthria, dysphagia, stridor, obstructive sleep apnoea, gastrointestinal (GI) or genitourinary (GU) dysfunction, parkinsonism, and comorbid neurological diseases. Gait impairment included falls, requiring gait aids or wheelchair dependence. GI/GU dysfunction included urinary retention, urinary incontinence, requirement for a urinary catheter and constipation.
The content of palliative care discussions was recorded if documented in the medical record. Topics entered in the analysis were discussions regarding diagnosis, symptom management, prognosis, goals of care (patients' wishes regarding life-extending measures, including resuscitation status, mechanical ventilation, artificial nutrition and level of care-e.g., no intensive care), comfort measures, ${ }^{7}$ decisions about DNAR, identification of substitute decision makers and decision making regarding tracheostomy. In most cases, there was limited explicit documentation surrounding the content of palliative care discussions; as such, any discussion involving diagnosis, symptom management, prognosis and goals of care was considered to be a palliative care discussion. Since the purpose of our study is to identify gaps in healthcare provision, we classify such discussions as palliative in order to avoid reporting false negatives. The number of discussions that would have been considered to be palliative by all parties may be lower than what is reported here. Similarly, cases in which discussions were not documented in the medical record were classified as "unknown."

\section{Sources of Bias}

To reduce bias, only data documented in the charts were included in the analysis. It is generally known that some advance care and palliative care discussions occur without documentation in patients' charts. ${ }^{8}$ Evidence of such discussions was not readily available, and review of this information with the treating neurologist would introduce recall bias, so the discussions not documented in the charts were excluded from the analysis.

\section{Statistical Analysis}

Due to the small number of patients included in the study and the descriptive nature of the data, a detailed statistical analysis could not be performed.

\section{ReSUlts}

A total of 26 patients were identified and screened, 22 of whom satisfied the inclusion and exclusion criteria. Four patients were excluded because there was not a clear clinical diagnosis of MSA. The demographic information for the 22 patients is outlined in Table 1 . Briefly, the mean age was $65.5 \pm 7.9$ years, with a mean age at time of diagnosis of $62.1 \pm 8.7$ years. The total symptom burden is depicted in Figure 1. All patients had parkinsonism: 12 were of the MSA-P subgroup and 10 were of the MSA-C

Table 1: Characteristics of patients with multiple system atrophy seen in outpatient neurology clinics or admitted under inpatient services at London Health Sciences Centre from January of 2004 to January of 2015

\begin{tabular}{|c|c|c|c|c|}
\hline & Total MSA (\%) & \multicolumn{3}{|c|}{ Those with stridor } \\
\hline All patients & & Total & No tracheostomy & Tracheostomy \\
\hline Female & $22(100 \%)$ & $6(27 \%$ of 22$)$ & $3(50 \%$ of 6$)$ & $3(50 \%$ of 6$)$ \\
\hline Male & $12(55 \%$ of 22$)$ & $3(25 \%$ of 12$)$ & $1(33 \%$ of 3$)$ & $2(67 \%$ of 3$)$ \\
\hline Age at diagnoses & $10(45 \%$ of 22$)$ & $3(3 \%$ of 10$)$ & $2(67 \%$ of 3$)$ & $1(33 \%$ of 3$)$ \\
\hline Age & $62.1 \pm 8.7$ & $60.8 \pm 7.2$ & $56 \pm 5.3$ & $65.6 \pm 5.7$ \\
\hline Duration of disease (mean in years) & $65.5 \pm 7.9$ & $64 \pm 6.4$ & $59.6 \pm 4.9$ & $68.3 \pm 4.9$ \\
\hline Deceased & $6.8 \pm 3.6$ & $6.4 \pm 4.4$ & $7.0 \pm 6.1$ & $5.5 \pm 0.7$ \\
\hline
\end{tabular}




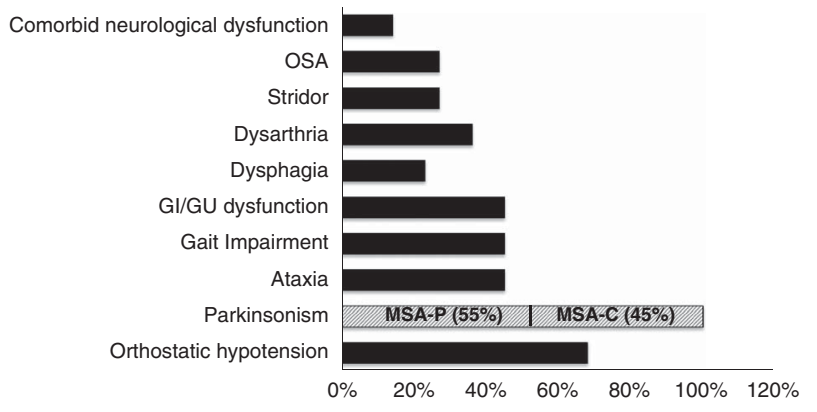

Figure 1: Total symptom burden of patients living with multiple system atrophy. The $x$ axis represents the total percentage of patients with that symptom, and the y axis represents the different symptoms.

subgroup. The next most common symptoms were orthostatic hypotension (15 patients), GI/GU dysfunction (10 patients), ataxia (10 patients) and gait impairment (10 patients).

\section{Sleep}

Seven patients underwent a sleep study. Of those, six were found to have obstructive sleep apnoea (OSA) without evidence of stridor, and one did not show sleep-disordered breathing but was later diagnosed clinically with stridor and had a laryngoscopy showing vocal cord paralysis. Three patients with confirmed OSA went on to develop vocal cord paralysis and stridor. In total, six patients were diagnosed with stridor, three of whom did not have a sleep study but were later found to have vocal cord paralysis by otolaryngology along with the three patients with previous OSA.

\section{Resuscitation Wishes}

Seven patients had formal documentation of a DNAR order in their chart, and one patient had it mentioned in a clinic note. One had a documented order to attempt resuscitation, and 13 patients had no evidence or documentation of code status in their medical record (duration of disease $=6.3 \pm 4.0$ years; see Table 2 ).

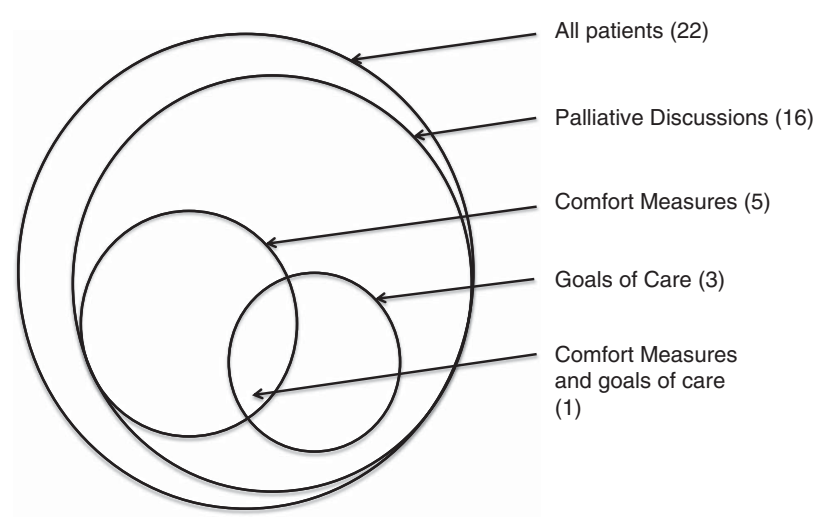

Figure 2: The different types of palliative care discussions taking place in multiple system atrophy. The categories of discussion topics investigated includes diagnosis and symptom management, goals of care discussions and comfort measures.

\section{Palliative Care Discussions}

Palliative care discussions, including those about symptom management, took place for 16 patients. Figure 2 outlines the nature of documented palliative care discussions. Of the 16 patients who had palliative care discussions (see Table 2 for timing), the most common topics were diagnosis, symptoms or symptom management, and prognosis. Of the patients who died (6), four had discussions about goals of care and decisions for comfort care made in the ICU prior to death, but no such discussions appear to have taken place prior to admission to the ICU. Of the group of patients still living (16), discussions surrounding goals of care occurred after an ICU admission and transfer to a complex care setting for one patient, and another patient had discussions during a multidisciplinary clinic for their concurrent motor neuron disease. The timing of discussions pertaining to goals of care from the time of diagnosis for these two patients was two years. Other discussions surrounding goals of care involved the need for artificial nutrition. Some 14 patients had documented dysphagia, 12 of whom had documented swallowing assessments. Six patients who had a

Table 2: Focus and timing of end-of-life discussions

\begin{tabular}{|c|c|c|c|c|}
\hline & \multirow{2}{*}{$\begin{array}{c}\text { MSA patients }(\%) \\
N=22\end{array}$} & \multicolumn{2}{|c|}{ Those with stridor $(n=6)$} & \multirow[t]{2}{*}{ Unknown } \\
\hline & & Tracheostomy $(n=3)$ & No tracheostomy $(n=3)$ & \\
\hline Respite care discussion & $6(27 \%$ of 22$)$ & $2(66 \%$ of 3$)$ & $1(33 \%$ of 3$)$ & $16(72 \%$ of 22$)$ \\
\hline Palliative care discussion & $16(72 \% \%$ of 22$)$ & $3(100 \%$ of 3$)$ & $1(33 \%$ of 3$)$ & $6(27 \%$ of 22$)$ \\
\hline Artificial nutrition discussion & $6(27 \%$ of 22$)$ & $1(33 \%$ of 3$)$ & $1(33 \%$ of 3$)$ & $15(68 \%$ of 22$)$ \\
\hline Appointed healthcare proxy & $7(32 \%$ of 22$)$ & $3(100 \%$ of 3$)$ & unknown & $15(68 \%$ of 22$)$ \\
\hline Do-not-attempt-resuscitation (DNAR) order & $8(36 \%$ of 22$)$ & $3(100 \%$ of 3$)$ & $1(33 \%$ of 3$)$ & $14(64 \%$ of 22$)$ \\
\hline Documented full code & $1(5 \%$ of 22$)$ & unknown & unknown & $14(64 \%$ of 22$)$ \\
\hline Total deceased & $6(27 \%$ of 22$)$ & $2(66 \%$ of 3$)$ & $1(33 \%$ of 3$)$ & 0 \\
\hline Time from admission to death (days) & hours $-28 \pm 18.2$ days & $24.5 \mathrm{~d} \pm 30.4 \mathrm{~d}$ & unknown & $1(16 \%$ of 6$)$ \\
\hline Time from palliative discussion to death (days) & hours -6 months & hours/16 days & 6 months & $1(16 \%$ of 6$)$ \\
\hline Documented DNAR in place prior to death & $2(33 \%$ of 6$)$ & $0(0 \%$ of 2$)$ & $1(33 \%$ of 3$)$ & $1(16 \%$ of 6$)$ \\
\hline Time from DNAR to death & hours -6 months & Hours/16 days & 6 months & $1(16 \%$ of 6$)$ \\
\hline Decision for DNAR made in emergency/intensive care unit prior to death & $4(66 \%$ of 6$)$ & $2(100 \%$ of 2$)$ & unknown & $1(16 \%$ of 6$)$ \\
\hline
\end{tabular}


swallowing assessment had recommendations for diet modifications to lower the risk of aspiration. One of these patients had a nasogastric tube in place during an ICU admission but did not require further enteral feeding after discharge. The remainder of the patients (five) with modified diets did not have a documented discussion about possible future requirements for artificial feeding. The other six patients were found to be severe aspiration risks and took part in subsequent discussions about decisions regarding enteral feeding (Table 2): five decided against and one elected to allow it.

\section{Stridor}

Six patients developed stridor, three of whom went on to have a tracheostomy (mean disease duration $=5.7 \pm 0.58$ years). Five of the six patients with stridor had a documented discussion about tracheostomy before one was required for life-sustaining purposes. Of the three patients who required emergent tracheostomy, one had a documented discussion declining tracheostomy in the management of stridor. Nevertheless, this patient was later intubated in intensive care secondary to respiratory failure. The patient's substitute decision makers decided on a tracheostomy after the patient could not be weaned from mechanical ventilation. Another patient had findings of sleep-disordered breathing but no documentation of discussions surrounding stridor or a potential need for tracheostomy in the future. This patient later presented to the emergency department with severe respiratory distress. Six attempts at intubation were made, and then an emergency tracheostomy was placed. This patient was later found to have vocal cord paralysis. A third patient had a discussion about the potential requirement for a tracheostomy in the future; however, there was no documentation regarding the patient's wishes prior to requiring a tracheostomy emergently.

\section{Deceased Patients}

Of the 22 patients, 6 were deceased. Two of the deceased (2/6) patients had a DNAR in place prior to death, one died at home (DNAR 6 months prior to death), and one (timing of DNAR uncertain because it was not documented in available records) had resuscitation attempted despite the DNAR. This occurred in the emergency department subsequent to an out-of-hospital cardiac arrest. The patient was found unresponsive at the nursing home where they had been living for three months, and resuscitation was initiated despite previously documented wishes. Further details are not available. There was clear evidence of an appointed substitute decision maker for only these two patients $(2 / 6)$ prior to time of death. Four of the deceased patients (4/6) had a DNAR decision made by a substitute decision maker during an ICU admission, during which it was determined that significant recovery was unlikely. Of this group (4 patients), two (2/4) had stridor, one (1/4) required an emergency tracheostomy, and the other (1/4) required a tracheostomy in the ICU due to ongoing requirements for mechanical ventilatory support. Of the four patients who died during their ICU admission, discussions surrounding resuscitation and goals of care took place 16 days prior to death in one patient and only hours before death in the other three.

\section{DISCUSSION}

Respiratory dysfunction, including but not limited to stridor, is the leading cause of death for people living with MSA, ${ }^{9}$ and it may present acutely with or without prior evidence of stridor. Early and effective advance care and end-of-life planning discussions are therefore essential for effective palliative care in MSA. Our chart review shows clear gaps in the current approach to advance care planning, goals of care and palliative care discussions in patients living with MSA, at least at our centre. Important advance care planning and end-of-life discussions took place very late in the disease trajectory and often at a time when the affected person was no longer able to participate. While our study is limited to a retrospective review of charts at a single centre, it nonetheless shows significant gaps in the provision of care.

The symptom profile of the study cohort was consistent with that reported in the literature. ${ }^{10}$ As expected, a minority $(27 \%)$ of patients developed stridor as a complication of MSA over the studied period. ${ }^{10,11}$ Our study found that specialists caring for patients with MSA recognize stridor as an important symptom and consider appropriate referrals and discussions surrounding tracheostomy. While discussions about tracheostomy were documented for most of the patients with stridor, patients' wishes regarding tracheostomy were not as consistently recorded. Research has previously suggested that continuous positive airway pressure may be beneficial in MSA patients with stridor. ${ }^{12}$ Anecdotally, our centre's experience with continuous positive airway pressure (CPAP) in MSA has not been as positive; difficulties have been noted with tolerance to CPAP. Our results show that physicians effectively engaged patients in discussions about diagnosis, symptom management and prognosis in MSA but were less thorough in documenting or engaging in discussions about DNAR and end-of-life planning. For half of the deceased individuals, DNAR and end-of-life discussions occurred just mere hours prior to death. One person had a prolonged hospital course in intensive care prior to any documented end-of-life discussions.

There is currently very little palliative care literature specific to MSA. However, research on such other neurodegenerative illnesses as Parkinson's disease (PD), motor neuron disease, Alzheimer's disease and multiple sclerosis ${ }^{13}$ supports the value of palliative care practices. For example, advanced care planning has been found to improve outcomes with respect to the dying process, to increase the satisfaction of family members with the dying process, to reduce the burden on caregivers and to reduce overall healthcare costs. ${ }^{14}$ Moreover, studies have shown that patients engage in end-of-life discussions and planning at the time of diagnosis when they are aware of having a chronic or progressive condition. ${ }^{14}$ Overall, people living with PD preferred to discuss treatment earlier in the disease course and wanted family members to be involved early on, while only half of patients wished to defer discussing end-of-life planning until their condition had worsened. $^{15}$

Unfortunately, there is little available evidence in the literature that explores when and how to initiate a comprehensive discussion about the associated risks and benefits of therapies in MSA. As our study shows, the provision of palliative care to people living with MSA could be improved through more timely and more comprehensive palliative discussions. Early and effective discussions about end-of-life care will allow people with MSA to make treatment decisions that better align with their goals of care prior to an emergent situation. We propose a framework to facilitate palliative care discussions for patients living with MSA and their healthcare teams. 


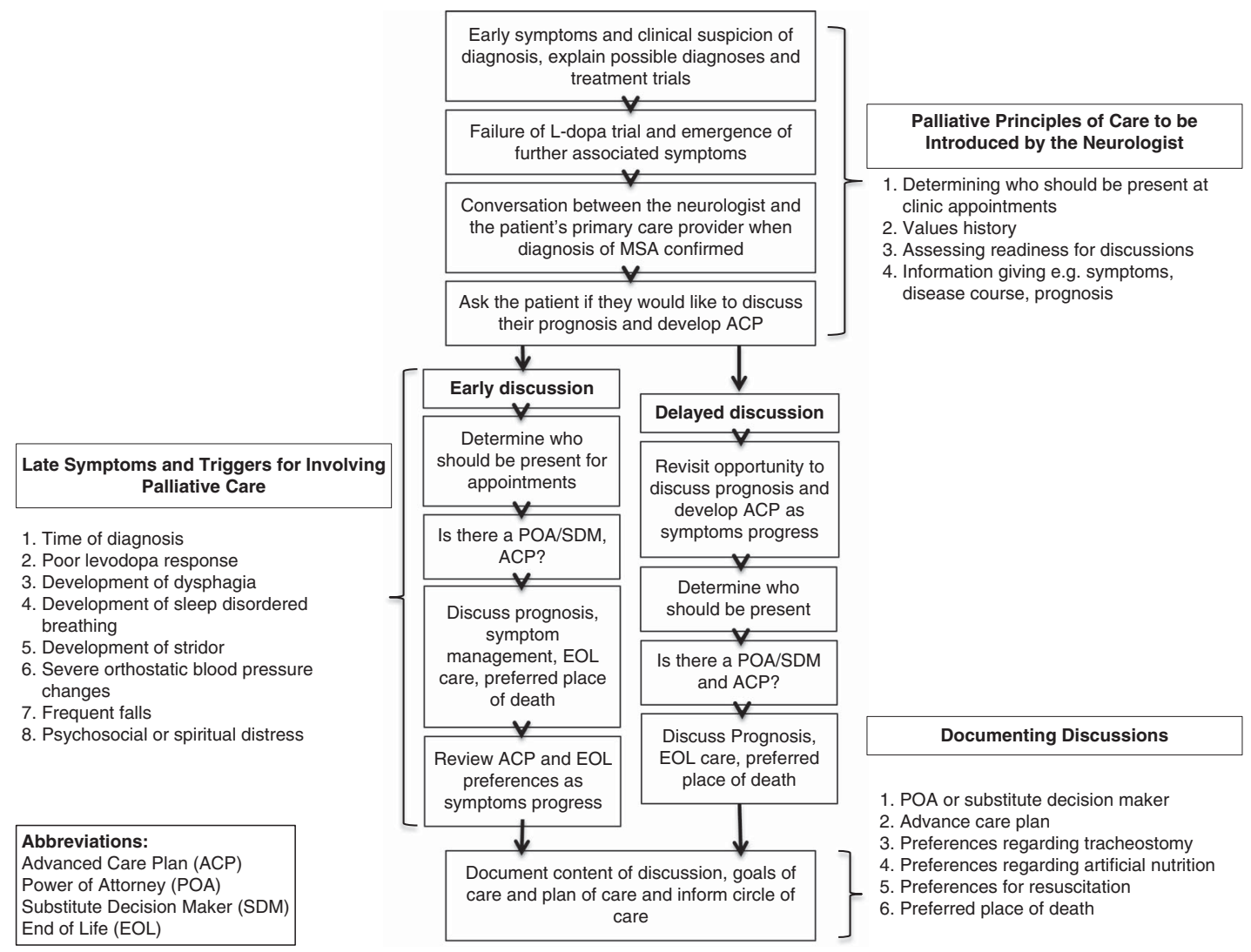

Figure 3: Proposed framework for palliative care discussions in MSA patients.

\section{Proposed Framework for Palliative Care Discussions in MSA}

The benefits of a palliative approach in the care of people living with incurable neurodegenerative disease have been shown elsewhere. ${ }^{15-17}$ Regardless of the approach taken to palliative care, there are certain key elements that should be a part of any discussions for people living with MSA, even before presenting with symptoms of respiratory dysfunction. Figure 3 outlines a proposed framework for a palliative approach to care for people with MSA. This framework reflects the general principles of palliative care and suggests how they could be applied in the case of MSA. In the early stages of MSA, it is important to determine who the person with MSA would like to have present for important healthcare discussions in general, and then establish each patient's readiness to discuss end-of-life decisions. Since diagnosis of MSA is difficult, there may be a period of ambiguity regarding the diagnosis, and the patient might not yet be receptive to hearing about the disease trajectory and his/her prognosis. Nevertheless, even without a determinate diagnosis, identifying personal supports and discussing personal values will help to inform and simplify future discussions regarding end-of-life care, when appropriate. Information regarding diagnosis, expected disease trajectory, symptom management and prognosis should be disclosed to the person with MSA and his/her family or an appointed decision maker, where possible. Physicians should initiate and document the results of important discussions, after assessing the patient's readiness for such discussions. These include the identification of a substitute decision maker or power of attorney for healthcare, the presence of advance care plans, preferences regarding artificial nutrition and hydration, including their expected benefits or the lack thereof, end-of-life care wishes and preferred place of death. While tracheostomy may extend the length of life, it does not, in our view, extend or improve the quality of life. Therefore, it is important to discuss tracheostomy and to document the patient's wishes surrounding tracheostomy before an unexpected emergent event.

Although there are no clearly identified triggers for referral to more specialized palliative care services, the evidence suggests that a diagnosis of MSA-P and the presence of urinary retention are clinical predictors of a poor outcome. ${ }^{10}$ Predictors of a more precipitous functional decline include a shorter duration from symptom onset and a poor levodopa response from the outset. ${ }^{10}$ Several other events during the disease trajectory might also provide a trigger for referral to palliative care services. For some people, a referral at the time of diagnosis might be appropriate, whereas for other people it may be more appropriate to initiate a referral once further severe symptoms develop, including dysphagia, stridor, sleep-disordered breathing, severe orthostatic blood pressure changes, increasing falls or other symptoms of distress.

Neurologists are usually the primary physicians managing patients with MSA throughout the disease trajectory. Although MSA is a rare condition, the field of neurology has a number of progressive neurological conditions, and as such it is appropriate for neurologists to be active participants in the palliative care process. Our study suggests that there are gaps in the palliative care provided in MSA, which may reflect the gaps in general neurology training suggested in the literature. Miyaski and Kluger ${ }^{18}$ describe a framework for palliative care in neurology with the aim of relieving the many facets of suffering, with a focus on the family as the unit of care. While it is 
important for neurologists to have the skills necessary for initiating palliative care discussions and end-of-life care, involving a multidisciplinary team including palliative care physicians or palliative care teams may allow for a more collaborative and holistic approach to care in advanced MSA. Neurologists with palliative care skills and palliative physicians with knowledge of rare neurodegenerative diseases may both contribute to improved patient care in MSA.

\section{Limitations OF THE STUdY}

This is a single-centre cohort study based on a retrospective review of a small number of cases $(n=22)$. It is possible that our centre or the considered cohort is not representative of the general population. However, because MSA is an uncommon disorder and its diagnosis is difficult, there are many barriers to larger-scale studies, and this is reflected in the small amount of published research on the topic. Moreover, prospective studies and controlled-variable studies can be difficult to implement in a palliative care setting for ethical reasons. Given the difficulty of studying uncommon disorders, we believe that both the size and scope of the study are appropriate to the objective of identifying gaps in the delivery of care. Moreover, since MSA is most likely diagnosed and treated at an academic centre aiming to provide best practices, our results are likely representative of other centres in Canada and North America and, at the very least, shed light on an important question for further investigation in a larger study.

The nature of our study also does not allow for a detailed analysis of the content and timing of end-of-life discussions. Prior studies have shown that physicians' recordkeeping does not reveal the depth and quality of palliative discussions. ${ }^{8}$ On the other hand, results from deceased patients in our study show that discussions about important decisions for tracheostomy and DNAR are often not made until an urgent situation. While patient records do not provide a perfect representation of actual discussions, this deficiency itself argues for the value of a framework for palliative discussions and recordkeeping.

\section{ConClusions}

Our study demonstrates that there is no standard approach to advanced care planning and palliative care discussions for people living with MSA at our centre. A systematic approach to palliative care in MSA would enable people living with MSA and their substitute decision makers to make healthcare decisions that are aligned with their healthcare goals and their wishes for care at the end of life. We propose a framework for advance care planning and palliative care discussions in MSA in order to help guide healthcare teams through these difficult but vitally important discussions.

\section{ACKNOWLEDGMENTS}

Our thanks go out to B. Tyson Gofton for editorial expertise and support.

\section{Disclosures}

Anita Dayal, Mary Jenkins, Mandar Jog, Kurt Kimpinski, Penny MacDonald and Teneille Gofton hereby state that they have nothing to disclose.

\section{Statement OF Authorship}

Anita Dayal participated in conceptualizing the study, interpreting the data, and drafting the manuscript. Mary Jenkins contributed to interpretation of the data and revising the manuscript. Mandar Jog contributed to interpretation of the data and revision of the manuscript. Kurt Kimpinski contributed to interpretation of the data and revision of the manuscript. Penny MacDonald contributed to interpretation of the data and revision of the manuscript. Teneille Gofton participated in conceptualization, data interpretation, and drafting and revising of the manuscript. The statistical analysis was performed by Drs. Anita Dayal and Teneille Gofton.

\section{REFERENCES}

1. Parkinsonian syndromes. In: Bradley RB, et al., editors. Neurology in Clinical Practice. Philadelphia: Butterworth Heinemann; 2012: P. 2230-1.

2. Silber MH, Levine S. Stridor and death in multiple system atrophy. Mov Disord. 2000;15(4):699-704.

3. Hardy J. Multiple system atrophy: pathophysiology, treatment and nursing care. Nurs Stand. 2008;22(22):50-6; quiz 58.

4. Yamaguchi M, Arai K, Asahina M, Hattori T. Laryngeal stridor in multiple system atrophy. Eur Neurol. 2003;49(3):154-9.

5. Wenning GK, Geser F, Poewe W. Therapeutic strategies in multiple system atrophy. Mov Disord. 2005;20(Suppl 12): S67-76.

6. Gilman S, Wenning GK, Low PA, Brooks DJ, Mathias CJ, Trojanowski JQ, et al. Second consensus statement on the diagnosis of multiple system atrophy. Neurology. 2008;71(9):670-6; Available at: https://www.ncbi.nlm.nih.gov/pmc/articles/PMC2676 993/pdf/5745.pdf.

7. Speak Up (2016). Glossary: Comfort Measures. Ottawa: Canadian Hospice Palliative Care Association. Available at: http://www.advancecareplanning.ca/resource/advance-care-planning-terms/\#1442266794389-239b6bb9-7a46.

8. Cox K, Moghaddam N, Almack K, Pollock K, Seymour J. Is it recorded in the notes? Documentation of end-of-life care and preferred place to die discussions in the final weeks of life. BMC Palliat Care. 2011;10:10-8; Available at: https://www.ncbi. nlm.nih.gov/pmc/articles/PMC3227605/pdf/1472-684X-1018.pdf.

9. Schrag A, Wenning GK, Quinn N, Ben-Shlomo Y. Survival in multiple system atrophy. Mov Disord. 2008;23(2): 294-6.

10. Wenning GK, Geser F, Krismer F, Seppi K, Duerr S, Boesch S, Köllensperger $\mathrm{M}$, et al. The natural history of multiple system atrophy: a prospective European cohort study. Lancet Neurol. 2013;12(3):264-74; Epub ahead of print Feb 5. Available at: https://www.ncbi.nlm.nih.gov/pmc/articles/PMC3581815/.

11. Wenning GK, Tison F, Ben Shlomo Y, Daniel SE, Quinn NP. Multiple system atrophy: a review of 203 pathologically proven cases. Mov Disord. 1997;12(2):133-47.

12. Iranzo A, Santamaria J, Tolosa E, Vilaseca I, Valldeoriola F, Martí MJ, et al. Long-term effects of CPAP in the treatment of nocturnal stridor in multiple system atrophy. Neurology. 2004; 63(5):930-2.

13. Gofton TE, Jog MS, Schulz V. A palliative approach to neurological care: a literature review. Can J Neurol Sci. 2009;36 (3):296-302.

14. Almack K, Cox K, Moghaddam N, Pollock K, Seymour J. After you: conversations between patients and healthcare professionals in planning for end of life care. BMC Palliat Care. 2012;11: 11-5; Available at: https://www.ncbi.nlm.nih.gov/pmc/articles/ PMC3517317/pdf/1472-684X-11-15.pdf.

15. Tuck KK, Brod L, Nutt J, Fromme EK. Preferences of patients with Parkinson's disease for communication about advanced care planning. Am J Hosp Palliat Care. 2015;32(1):68-77; Epub ahead of print Sep 19, 2013. Available at: http://journals.sagepub.com/ doi/pdf/10.1177/1049909113504241. 
16. Chahine LM, Malik B, Davis M. Palliative care needs of patients with neurologic or neurosurgical conditions. Eur J Neurol. 2008;15(12):1265-72.

17. Dawson S, Kristjanson LJ. Mapping the journey: family carers' perceptions of issues related to end-stage care of individuals with muscular dystrophy or motor neurone disease. J Palliat Care. 2003;19(1):36-42.

18. Miyasaki JM, Kluger B. Palliative care for Parkinson's disease: has the time come? Curr Neurol Neurosci Rep. 2015;15 (5):26. 